\title{
The Impact of Regulated Cell Death Pathways on Alloimmune Responses and Graft Injury
}

\author{
Marie-Josee Hébert ${ }^{1,2}$ • Anthony M. Jevnikar ${ }^{2,3}$
}

Published online: 4 July 2015

(C) Springer International Publishing AG 2015

\begin{abstract}
Transplantation is invariably associated with organ injury following donor organ procurement. Death of cells can negatively impact organ function if sufficient numbers of parenchymal cells are eliminated and not replaced as part of remodelling. Additionally by the release of contents, the death of cells can alter immune responses that are related to ischemia-reperfusion injury and alloimmune rejection. There is increasing awareness of the link between innate and adaptive immunity, and the profound influence inflammation has on organ function, tolerance induction, rejection responses and perhaps survival. Unfortunately, long-term survival of transplants has not been greatly impacted by advances in transplant strategies that solely target recipient immune responses. Therefore, a focus on better understanding tissue injury, and characterizing how newly described forms of cell death influence inflammation in transplanted organs, is timely and likely to yield more effective strategies. Until recently, programmed cell death was relegated entirely to apoptosis, a form of death that is largely, but not exclusively, silent immu-
\end{abstract}

This article is part of the Topical Collection on Immunology

Anthony M. Jevnikar

jevnikar@uwo.ca

1 CRCHUM, Université de Montréal Multi-Organ Transplant Program, Université de Montréal, Montréal, Québec, Canada

2 Canadian National Transplant Research Program (CNTRP)

3 Matthew Mailing Centre for Translational Transplant Studies, Multi-Organ Transplant Program, London Health Sciences Centre, University of Western University, London, ON, Canada nologically. In contrast, necrosis is highly pro-inflammatory, but has not been considered to be regulatable, and is thus beyond therapeutic targeting. This partitioning of cell death has become blurred as regulated forms of necrosis such as necroptosis have been identified and immune consequences of apoptosis are characterized. While these share in a common capacity to promote inflammation, they vary considerably in specific pathways, susceptibility to therapeutic intervention, and indeed, in specific organ relevance. This review will discuss current understanding of apoptosis and regulated forms of necrosis in organ injury with specific reference to ischemiareperfusion injury and allogeneic solid organ transplantation.

Keywords Apoptosis $\cdot$ Necroptosis $\cdot$ Programmed cell death $\cdot$ Transplantation $\cdot$ Inflammation

\section{Introduction}

The concept of programmed cell death, although dating back to the late nineteenth century, really came to 'life' in the 1990s with the characterization of molecular pathways regulating the various phases of apoptosis in mammalian cells. Apoptosis, the only type of programmed cell death known at the time, was found to be controlled by precise molecular programs that initiate and transduce death signals towards cellular execution. At the time, apoptosis, a process that partitioned cellular contents within membrane vesicles following death, was described in stark contrast to necrosis, which was considered to be an unregulated type of cell death associated with cell swelling, cell membrane permeabilisation and rupture. The latter form of death, in contrast to apoptosis, resulted in the massive release of danger-associated molecular patterns (DAMPs) to ignite rapid and robust inflammatory responses. 
Apoptosis was morphologically associated with asymmetric chromatin condensation (marginalization), global nuclear condensation (pyknosis), nuclear fragmentation (karyorrhexis), production of membrane-bound blebs referred to as apoptotic bodies, and absence of cell membrane permeabilisation or rupture - thus without DAMP release [1]. As such, apoptosis was thought to go 'unnoticed' by the immune system, or even to be anti-inflammatory.

Life and (cell) death have become much more complicated and dogmas dating back many decades have now been challenged. The immunologically 'silent' nature of caspasedependent apoptosis has been challenged, and mounting evidence suggests a link between apoptosis, rejection and allograft survival. As well, new forms of regulated cell death have been described, including forms that induce morphological changes identical to classical necrosis. Molecular pathways controlling regulated necrosis, and in particular, 'necroptosis', have now been well characterized, and importantly highlight a potential for pharmacological inhibition of this form of cell death as well as apoptosis in transplantation. Here, we will review the challenges and promises for transplantation of identifying and perhaps harnessing cell death pathways to better control allograft injury with both ischemia-reperfusion $(\mathrm{I} / \mathrm{R})$ and alloimmune rejection.

\section{Apoptosis Dysregulation in Transplantation}

Transplantation is associated with enhanced numbers or maladapted function of apoptotic cells, either within resident parenchymal cells or infiltrating leukocytes, or most often, in both. A better understanding of the role of apoptosis in allograft dysfunction calls for attention to all types of cells undergoing apoptosis, the sustainability of the apoptotic response within the allograft, and the response of the immune system to an increased apoptotic load, which can, in itself, modulate immune recognition. It is generally accepted that apoptosis can be initiated by two major classes of death triggers: death receptors or mitochondrial outer membrane permeabilisation (MOMP) [2] (Fig. 1).

Mitochondrial destabilization leading to intrinsic apoptosis can be induced by various stressors, many of which are present during organ preservation and ischemia/reperfusion (I/R) (hypoxia, reactive oxygen species, growth factor deprivation and accumulation of misfolded proteins). This leads to the accumulation of pore-forming, pro-apoptotic members of the $\mathrm{Bcl}-2$ protein family such as BAK and BAX at the mitochondrial level, resulting in MOMP and cytosolic release of cytochrome $\mathrm{C}$ and other pro-apoptotic mitochondrial molecules [apoptosis-inducing factor (AIF), endonuclease G (ENDOG), direct IAP-binding protein with low pI (DIABLO/SMAC,) and high temperature requirement protein A2 (HTRA2)] [2]. Upon cytosolic relocalisation, cytochrome $\mathrm{C}$ interacts with
APAF1 (Apoptotic peptidase activating factor 1) and dATP, therefore forming an 'apoptosome', a multiprotein complex responsible for cleavage of effector caspases 3,6 and 7. Activation of effector caspases initiates classical morphological changes that are due, at least in part, to caspase-dependent degradation of nuclear and cytoskeletal substrates.

Increased activation of effector caspases has been reported in models of I/R injury in kidneys, liver and lung (Table 1). In models of renal $\mathrm{I} / \mathrm{R}$, small interfering RNAs or shRNAs targeting caspase-3, used intravenously or in the perfusion fluid, reduced renal cell death, inhibited inflammation and prevented accumulation of extracellular matrix [3, 4]. Interestingly, concomitant silencing of both caspase- 3 and caspase- 8 (see below) showed enhanced renal protection [5], an important issue as we have learned more about the role of capase- 8 in regulating necroptosis in some cell types. Caspase- 3 silencing prior to I/R also improved lung function and reduced pulmonary cell death in rats [6]. In a model of liver transplantation in rats, inhibition of caspase- 3 and caspase- 7 with the biochemical inhibitor DEVD-FMK in the donor and in the preservation fluid led to reduced microvascular injury and improved survival post-transplant [7]. Intriguingly, in a model of heart transplantation in mice, administration of a caspase- 3 inhibitor in both the donor and recipient before transplantation led to reduced coronary artery vasculopathy (CAV) [8], suggesting that caspase-dependent vascular damage at the time of transplantation may fuel maladaptive immune responses implicated in progressive vascular obliteration. In support of this hypothesis, hearts from transgenic mice overexpressing the anti-apoptotic gene Bcl-2 under the control of myosin heavy chain promoter transplanted into wild-type mice exhibited less inflammation and reduced coronary artery vasculopathy (CAV) [9]. Another report, however, using adenoviral vectors for induction of Bcl-2 overexpression in the heart before transplantation, described reduced heart apoptosis in the first days post-transplantation, yet enhanced CAV in the long term [10]. These seemingly contradictory results might be explained, at least in part, by the different methods of $\mathrm{Bcl}-2$ overexpression used in the donor heart, likely contributing to induction of BCL-2 expression in different cell types. Whether BCL-2 vectors administered by intracoronary injection at the time of transplantation could have also transduced immune cells and enhanced survival of rejection effectors was not evaluated. Finally, in an animal model of liver transplantation, pancaspase inhibitors administered during liver perfusion and cold storage led to improved liver function [11]. Similar findings were reported in human liver transplantation preserved during cold storage in the presence of a pan-caspase inhibitor [12]. Clinical studies, currently underway or completed, are testing the safety and/or efficacy of caspase inhibitors in islet transplantation (ClinicalTrials.gov Identifier: NCT01653899) and liver transplantation (ClinicalTrials.gov Identifier: NCT00080236). 


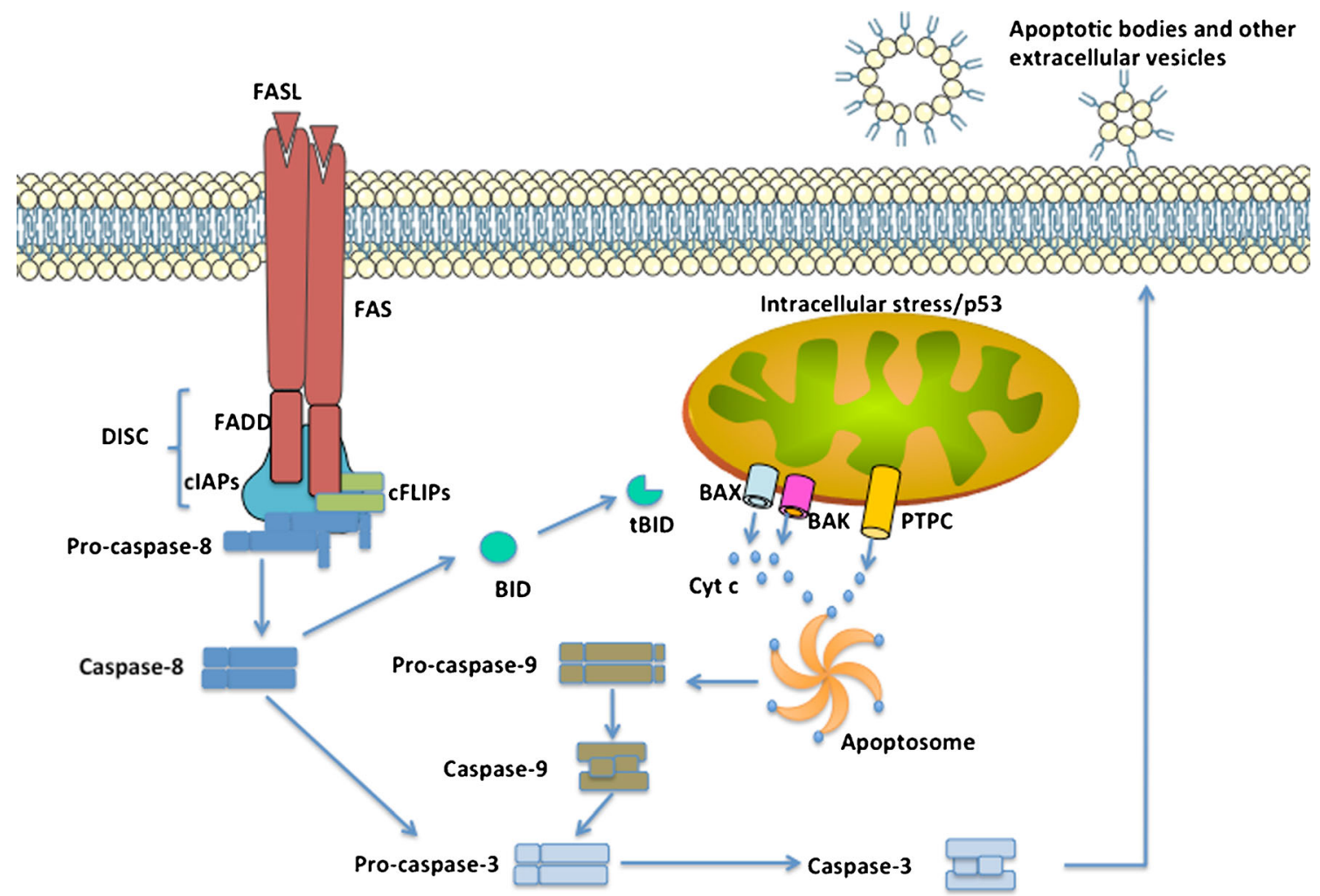

Fig. 1 Extrinsic apoptosis is triggered by interactions between death receptors and their ligand (here exemplified by FAS/FASL), leading to assembly of a death-inducing complex (DISC) composed of the cytoplasmic tail of the death receptor (FAS), FAS-associated protein with a death domain (FADD), cellular inhibitor of apoptosis proteins (cIAPs), c-FLIPs and pro-caspase- 8 . The DISC controls the cleavage and activation of caspase-8, leading directly to activation of effector caspases (such as caspase-3) or to mitochondrial permealisation secondary to BID cleavage. Intrinsic apoptosis is induced by

The tumor suppressor gene $\mathrm{p} 53$ is an important regulator of mitochondrial apoptosis. p53 is stabilized in response to stress, therefore favouring interactions with pro-apoptotic members of the Bcl-2 family, mitochondrial translocation and MOMP $[13,14]$. p53 also regulates the propensity to die by apoptosis by activating the transcription of various pro-apoptotic genes, including Bax, Bid, Noxa and Puma [2]. Over the past 10 years, various reports suggested a beneficial role for p53 inhibition in preventing $\mathrm{I} / \mathrm{R}$ injury. Biochemical p53 inhibition with pifithrin- $\alpha$, gene knock-out and small interfering RNAs targeting p53 have generally been associated with reduced parenchymal apoptosis and improved organ function following I/R injury when administered acutely [11, 15-20] (Table 1). Prolonged administration, however, was associated with worsened renal function and increased fibrosis post-I/R. Long-term pifithrin- $\alpha$ administration led to enhanced extracellular matrix production, epithelial-tomesenchymal transition (EMT), and amplified inflammatory responses [18]. Worsened renal function post-I/R has also been reported in p53 -/- mice [21]. This detrimental effect was also observed with leukocyte-specific p53 invalidation, intracellular stressors that stabilize p53, facilitate mitochondrial translocation of BAX and BAK and opening of the permeability transition pore complex (PTPC). This leads to the release of various mediators, including cytochrome $\mathrm{C}$ (Cyt c), that react with APAF-1 in the presence of dATP to form an apoptosome. The apoptosome activates caspase-9, which in turn cleaves and activates effector caspases such as caspase-3. Caspase-3 regulates the release of various types of membranebound vesicles by the apoptotic cell in the absence of cell membrane permeabilisation

suggesting an important role for p53 in controlling immune responses following injury. In support of this, p53 -/- mice exposed to $\mathrm{I} / \mathrm{R}$ showed reduced numbers of antiinflammatory M2 macrophages. These results speak to the importance of assessing the role of cell death interruption in specific cell types, as global invalidation of a given death regulator in allograft parenchymal cells could acutely prevent cell death in situ, but in the long-term could deregulate immune responses leading to sustained immune attack and worsened organ injury. A clinical phase I/II study in patients who are undergoing renal transplantation from deceased donors is underway to evaluate the safety and pharmacokinetic profile of a siRNA-targeting p53 (ClinicalTrials.gov Identifier: NCT00802347).

Apoptosis induced by extracellular ligands that interact with specific transmembrane receptors is referred to as 'extrinsic apoptosis' [2]. Classical lethal ligands such as FAS/ CD95 ligand (FASL/CD95L), tumor necrosis factor $\alpha$ $(\mathrm{TNF} \alpha)$ and TNF-related apoptosis-inducing ligand (TRAIL), bind to FAS/CD95, TNF $\alpha$ receptor 1 (TNFR1) and TRAIL receptor (TRAILR) 1-2, respectively. These interactions 


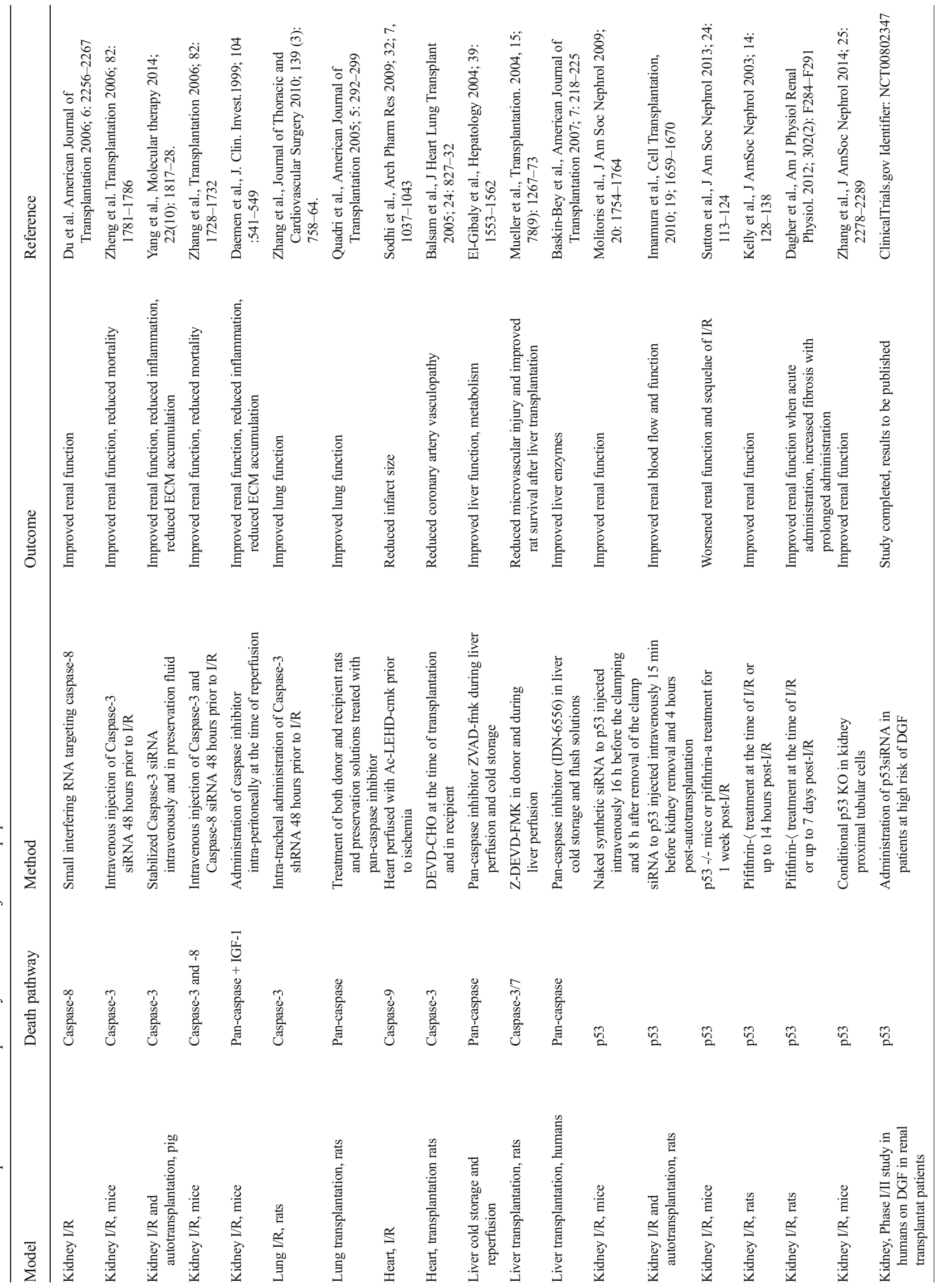




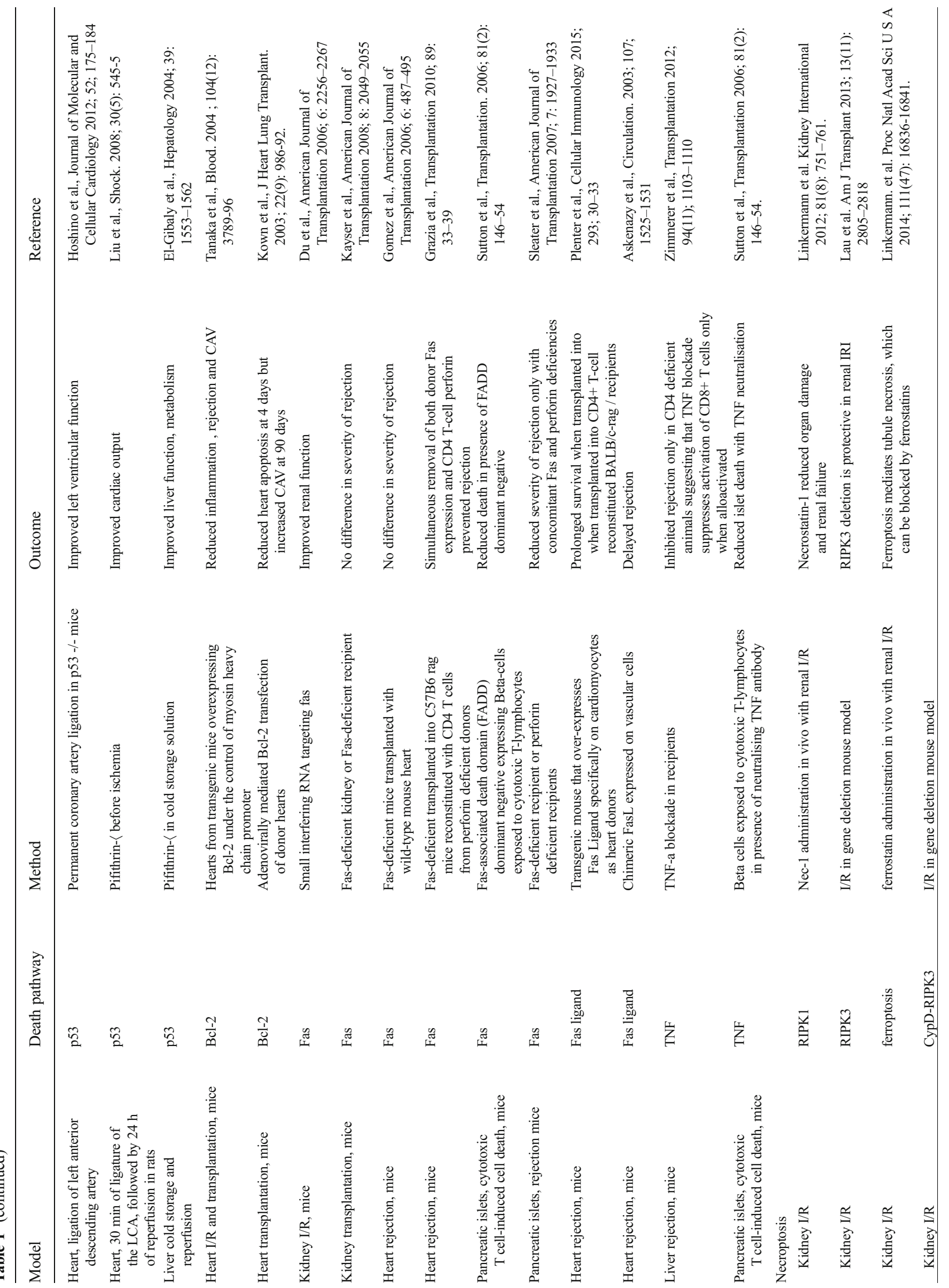


prompt the recruitment of a multiprotein complex at the cytoplasmic death domain of the receptor [referred to as the deathinducing signalling complex (DISC)], acting as a platform for caspase-8 activation [2] (Fig. 1). In some cells, activated caspase- 8 cleaves caspase-3 directly, therefore triggering the execution phase of apoptosis. In other cell types, however, caspase- 8 activation leads to MOMP through cleavage of BH3-interacting domain death agonist (BID), leading to the generation of a truncated fragment (tBID) with mitochondrion-permeabilising activity. Apoptosis can also be induced by a distinct truncated BID fragment in cells targeted by cytotoxic $\mathrm{T}$ cells or natural killer cells and exposed to the granzyme/perforin system [22]. Upon interaction with target cells, granzyme B is delivered through perforindependent pathways, leading to cleavage of BID and induction of MOMP. Granzyme B and other granzymes can also activate caspase-3, leading to a retro-action loop on the mitochondria, MOMP and full activation of the mitochondrial apoptotic pathway $[23,24]$. Activated $\mathrm{T}$ cells can also induce apoptosis through granzyme B-dependent Bim activation favoring $\mathrm{Bak} / \mathrm{Bax}$ interactions and mitochondrial destabilisation [25]. The granzyme B-perforin system plays an important role in allograft cell death triggered by cellular rejection. Granzyme B and perforin transcripts are increased in association with acute rejection of renal, liver, pancreatic and heart allografts [26-29]. Loss of SPI-6 (a homologue of human PI9 ), an endogenous inhibitor of Granzyme-B action in kidney cells, accelerated rejection and loss of function following transplant in a mouse model [30••]. The Fas-Fas ligand system also plays a major role in T-cell-mediated killing. Fas ligand expression increases on $\mathrm{T}$ cells following their activation, promoting deadly interactions with Fas on target cells. However, Fas ligand/Fas interactions also serve to control unopposed immune activation. T cells become sensitive to Fas-mediated apoptosis after their activation and undergo Fas-dependent activation-induced cell death (AICD). Abnormalities in the Fas ligand/Fas pathway in immune cells have been associated with exuberant immune responses and autoimmune phenotypes.

In transplantation, various teams have attempted to target the Fas-Fas ligand and/or perforin-granzyme B systems to inhibit rejection, I/R injury or both. Our group showed that shRNAs targeting Fas or caspase- 8 protected mouse kidneys from I/R injury, as demonstrated by improved renal function and reduced tubular epithelial cell apoptosis [31]. The importance of the Fas/Fas ligand and perforin/granzyme B systems in the rejection of renal allografts is, however, still debated. Although increased urinary perforin transcripts have been associated with rejection in human renal transplant patients [26], transplantation of Fas-deficient mouse kidneys into either perforin or granzyme B deficient recipients did not yield any reduction in rejection intensity (Table 1) [32]. Importantly, this was not in a survival transplant model and relied on gene 
expression analyses to show similar molecular disturbances in Fas-deficient and wild type allografts [32]. In contrast, using recipient mice with bilateral nephrectomies, both Fas and FasL deficient donor kidney transplants imparted a functional and survival benefit. Furthermore, it was shown that tubular epithelial cells express both Fas and FasL and can induce a form of 'fratricide' by engaging these molecules [33]. This suggests some caution in interpretation of results, as there are often differences in models. More importantly, this shows that regulation of apoptosis occurs within kidney parenchyma by the external co-expression of 'death' molecules, as well as internally by caspase regulation. Tubular epithelial cell (TEC) fratricide through Fas-FasL induced apoptosis has also been shown in cisplatin-induced kidney failure, suggesting that this is an active program in diverse forms of inflammatory TEC injury including transplantation, which may be obscured by diverse forms of death and the model selected [34]. In heart transplantation, concomitant inactivation of perforin/ granzyme B and Fas/Fas ligand systems was associated with mitigation of rejection. Fas-deficient hearts transplanted into C57B6/rag null recipients showed reduced rejection intensity only when recipients were reconstituted with perforindeficient CD4 T cells [35]. Interestingly, a reduction in rejection intensity was also reported with expression of chimeric Fas ligand on vascular cells [36]. Hearts from transgenic mice that over-expressed Fas Ligand on cardiomyocytes were protected from rejection after transplantation when transplanted into CD4+ T-cell-reconstituted BALB/rag recipients [37]. These data suggest Fas expressed on CD4 T cells can interact with Fas ligand on donor cardiomyocytes to modulate CD4 T cell function and/or survival. Pancreatic islets were also found to be protected from rejection when Fasdeficient islets were transplanted into perforin-deficient recipients, whereas deficiency in only one of these pathways in either the donor or recipient did not modulate rejection [28]. Collectively, these results suggest that some types of organ transplantation may benefit from concomitant inhibition of the Fas/Fas ligand and perforin/granzyme B pathways. However, the necessity to intervene at multiple levels and the potential independence from Fas for T-cell-mediated injury suggests that this strategy may be difficult to implement in transplantation by pharmacological interventions.

\section{"After-Death" Communication of Importance in Transplantation}

Recent evidence has suggested the intriguing possibility that apoptosis (or other forms of programmed death) in one organ can destabilize microvascular homeostasis and activate microvascular injury in distant organs [38•, 39, 40]. Kidney I/R injury was shown to increase lung microvascular apoptosis and to lead to abnormal pulmonary vascular permeability
$[38 \cdot, 39]$. Inhibition of caspase-activation at the time of renal $\mathrm{I} / \mathrm{R}$ injury prevented concomitant lung microvascular cell death. Inhibiting TNF-dependent signalling with the TNFreceptor blocker etanercept also significantly reduced renal I/ $\mathrm{R}$-induced lung microvascular apoptosis and preserved lung function. These results highlight the important and unexpected role of renal cell death in regulation of lung microvascular injury through caspase-dependent and TNF- dependent pathways. The precise molecular pathways and mediators that contribute to this maladaptive apoptotic organ cross-talk remain to be characterized, but as discussed below, also involve regulated forms of necrosis [41]. Mounting evidence from our group and others has shown that caspase activation leads to the release of various types of membrane-bound extracellular vesicles, in addition to classical apoptotic bodies [42•, 43]. Increased circulating levels of extracellular vesicles are associated with many inflammatory conditions, suggesting they may participate in distant organ cross-talk [44]. Extracellular vesicles have also been shown to play important roles in proteolytic processing regulating TNF activation, hinting further at their potential role in organ cross-talk during acute injury [45]. Although plausible, further studies are needed for establishing a clear role of extracellular vesicles in distant organ cross-talk following I/R injury. Nonetheless, this could open new areas of interventions in organ donors for interrupting such maladaptive organ cross-talk that appears to induce microvascular injury before organ procurement prior to transplant, and increase the risk of allograft dysfunction.

Another unexpected collateral damage of apoptosis in transplantation is the intriguing association between antibody-mediated (humoral) reactivity to components of apoptotic cells and reduced allograft survival. The presence of autoantibodies reactive to apoptotic cell pretransplantation was recently shown to be associated with reduced allograft survival in renal transplant patients [46••, 47]. Our group recently characterized autoantibodies targeting a C-terminal fragment of perlecan released during endothelial apoptosis as a risk factor of acute vascular rejection in renal transplant patients [48••]. Intriguingly, in our cohort of largely de novo transplant patients, these autoantibodies were present before transplantation. There was no association between levels of anti-perlecan/LG3 antibodies and classical autoimmune conditions. Passive transfer of anti-perlecan/LG3 antibodies in a model of vascular rejection in mice enhanced vascular inflammation and remodelling, demonstrating their role not only as predictors, but also as accelerators of rejection [48••]. The mechanisms that support loss of tolerance to components of apoptotic cells leading to the production of autoantibodies of importance in fuelling rejection remain largely uncharacterized. A better understanding of these pathways will provide much-needed insights into the autoimmune pathways that contribute to rejection. 


\section{Overview of Necroptosis, Regulated Necrosis and Transplant Injury}

Necrosis death of cells has long been considered to be a consequence of extreme or accidental stress, which in transplantation begins with the invariable injury associated with donor organ procurement and storage [49•]. As noted, this is followed by a complex biological program that further injures the organ, and is collectively referred to as I/R injury [50]. Parenchymal cells, including epithelial and endothelial cells in donor organs, are susceptible to many forms of death from metabolites from IR, including classical reactive oxidative species (ROS) and nitric oxide (NO), as well as pro-inflammatory cytokines, antibodies and cytotoxic effector cells that are central to alloimmune injury. Regardless of the form of injury, the subsequent death of cells by necrosis rather than apoptosis is associated with loss of membrane integrity and the subsequent release of pro-inflammatory intracellular contents collectively referred to as "cellular damage-associated molecular patterns" (CDAMPs) that promote inflammation [51, 52]. In transplantation, it is increasingly apparent that there is an impact of innate responses from infection and inflammation on tolerance induction, acute rejection and perhaps long-term graft survival [53]. Recently, there have been new insights into necrosis forms of death that have substantially altered our views on how cells regulate this form of death. In what may be a paradigm-changing discovery, subsets of necrosis are clearly regulated and apoptosis no longer exists as the sole form of programmed cell death [54-58]. This may be of particular significance to transplantation, as the identification of these pathways has already generated novel therapeutics such as necrostatins and ferrostatins, and others will follow. In the unique situation of transplantation, these might be delivered ex vivo to donor organs prior to transplant, or directly to recipients for short-term exposure during the early post transplant period. We will now review the area of regulated necrosis in relation to organ inflammation and transplantation. Given the current level of understanding in transplantation, the discussion will be confined to relevant pathways within the donor organ, although the recipient immune system is likely be influenced by modulation of regulated necrosis as well.

\section{What is Regulated or Programmed Necrosis?}

The discovery of regulated necrosis and ultimately necroptosis was based on the observation of caspaseindependent cell death in L929 cells exposed to TNF $\alpha$ along with the pan-caspase inhibitor zVAD-fmk [59]. While the anticipated result might have been reduced or absent apoptosis, surprisingly, cell death was enhanced and had morphologic features of necrosis. In relation to this, much of the previous work in cell death that distinguished apoptosis from necrosis has relied on morphology and structural/biochemical differences. Rather than typical cell shrinking, membrane blebs, membrane bound vesicles and condensation of chromatin in apoptosis, necrosis is associated with increased cell volume (oncosis), increasingly translucent cytoplasm, little chromatin condensation with intact nuclei, swelling of organelles, lysosomal membrane permeabilisation, and finally, disruption of the plasma membrane. This final step allows the leakage of cytoplasmic contents, which importantly are not membrane enclosed $[60 \bullet \cdot$. Without membrane enclosure, proinflammatory endogenous molecules, including heat-shock proteins (HSP), unprocessed high-mobility group box 1 (HMGB1), uric acid, fibronectin, IL-33 and others [61, 62], are free to interact with parenchymal and immune cells in the microenvironment of the transplanted organ. Of great recent interest, DAMPs from necrosis may also have more distant systemic effects, as do membrane bound vesicles following apoptosis. Cellular death associated molecular patterns (DAMPs) participate in I/R injury and allograft rejection, presumably through interaction with Toll Like Receptors (TLR), although other innate receptor signalling pathways exist [63-66]. As morphological features are generally shared between all forms of necrosis, they have not been particularly useful in differentiating between different forms of regulated necrosis. Indeed, there are also features shared with apoptosis that contribute to the problem of how best to differentiate cell death pathways. Annexin V staining, for example, is a commonly used in vitro method to detect phosphatidylserine (PS) that becomes exposed on the outer plasma membrane, thus representing early apoptosis. However, PS exposure can be detected in some necrotic cells that do not have disrupted plasma membranes [67]. Similarly, terminal deoxynucleotidyl transferase dUTP nick end labeling (TUNEL) staining used to detect DNA strand breaks in apoptosis can also detect breaks in necrotic cells [68]. A major obstacle in studying the role of regulated necrosis and necroptosis in vivo has been the lack of a definite molecular marker for the in situ identification of necroptotic cells. Following the initial observations supporting the existence of regulated forms of necrosis, there is now an intensive focus on more precise differentiation of necrosis, using molecular identification of the specific molecules involved, genetic disruption in animal models, and specific pharmacologic agents. Necrostatins (Nec-1), for example, have been used to identify necroptosis, although this approach also has pitfalls, as 'off target' effects influence other forms of necrosis or even perturb cellular homeostasis. This is particularly important in the clinic as newer 'death modifying' therapeutics become available, as disruption of such pathways without detailed biological understanding might result in beneficial or harmful outcomes [55].

The main focus of this review of regulated necrosis in transplantation is on RIPK1 and RIPK3-mediated necroptosis, as much of our current information is limited to this form of 
death in I/R injury organ injury and transplant survival. However, it is important to recognize that other potentially transplant relevant forms of regulated necrosis exist (reviewed in $[60 \bullet, 69])$. Mitochondrial permeability transition (MPT)-mediated regulated necrosis is related to cyclophilin D (CYPD), a key component of the permeability transition pore complex (PTPC). Interestingly, the widespread early use of cyclosporine may have improved early graft function through its effect on permeability transition pore related death and inflammation, and perhaps not entirely due to inhibition of $\mathrm{T}$ cell activation. Parthanatos is triggered by DNA-repair mechanisms and hyperactivation of poly(ADP-ribose) (PAR) polymerase 1 (PARP1) enzyme, which leads to cellular depletion of NAD+ and ATP energy. While parthanatos requires mitochondria, necroptosis can occur independently of mitochondria, which raises interesting questions as to potential strategies that target regulated necrosis in cold storage organs under anaerobic conditions $[70,71]$. We have recently observed a form of parthanatos in endothelial cells under inflammatory stress, but regulation by caspase and PARP inhibitors appears to be very sensitive to intracellular $\mathrm{pH}$ (Pavlovsky and Jevnikar, unpublished data). This observation may also provide some insight as to differences in previous reports regarding the worsening of transplant injury vs. attenuation of $I / R$ injury with caspase inhibition.

As in apoptosis, there is considerable 'cross-talk' between components involved in different forms of regulated necrosis. Besides its role in necroptosis, RIPK3 has also been implicated in facilitating pro-IL-1 $\beta$ processing in macrophages and dendritic cells. IL-1 $\beta$ is an innate inflammatory cytokine that requires cleavage and maturation by caspase- 1 , which occurs as a result of activation of a macromolecular complex termed the inflammasome. RIPK3 can promote NLRP3 inflammasome and IL-1 inflammatory responses independent of MLKL and necroptotic cell death [72]. Pyroptosisregulated necrosis occurs after inflammasome stimulation and has been shown to promote chronic kidney injury [66, 73]. Ferroptosis is a recently described caspase and necrosome-independent cell death form of regulated necrosis characterized by iron-dependent production of reactive oxygen species (ROS), increased levels of lipid hydroperoxides and inhibition by the iron chelator desferrioxamine (DFO) [69]. It can be induced by a number of small molecules named ferroptosis-inducing agents (FINs). It is important to note that ferroptosis can be blocked by Nec-1 demonstrating 'off-target' effects through as-of-yet unknown mechanisms [74]. Earlier studies of necroptosis based on responses to Nec-1 may thus have encompassed ferroptotic cell death as well. Indeed, it has recently been suggested that ferroptosis rather than necroptosis is the primary participant in kidney $I / R$ injury [75]. NETosis (neutrophil extracellular trap) is associated with release of DNA, chromatin and histones (NETs), which allows immune cells to kill microbial and viral infections. Autophagy is a crucial mechanism for cellular homeostasis, but under certain conditions, including disruption of other forms of cell death, it can become a cell-death mechanism itself. It is somewhat unique biochemically, as it is characterized by LC3 (microtubule-associated protein 1 light chain 3 ) and degradation of p62, a ubiquitin-binding scaffold protein. Interestingly, it can be promoted by the mTOR inhibitor sirolimus, and thus has a relationship to transplant drugs and some current models of necroptosis in transplantation.

\section{Triggering Necroptosis by Specific Signalling Pathways}

Necroptosis can be triggered through TNF receptor 1 (TNFR1) as well as other death receptors in the TNF superfamily, Toll-like receptor 3 (TLR3) and TLR4, and the interferon receptors [60••, 69] (Fig. 2). As noted earlier, there are six human death receptors (DRs) in the TNF superfamily: TNFR1, FAS (also known as CD95 or APO-1), DR3 (also known as TRAMP or APO-3), TRAILR1 (also known as DR4), TRAILR2 (also known as DR5, TRICK or KILLER), and DR6. It is noteworthy that TNFR1 signalling can diverge to either pro-survival or cell death, following complex proteinprotein interactions. In contrast, ligation of death receptors such as CD95/Fas or of TRAIL to TRAILR1 or TRAILR2 result in the formation of a Death-Inducing Signalling Complex (DISC), which by the association of the adaptor protein FADD, and recruitment and activation of caspase- 8 is essentially poised for apoptosis [76-78] Thus, there are differences in the early assembly of necroptosis components following receptor ligation $[54,79]$. The signalling pathway for necroptosis has been best characterized for TNF receptor 1 (TNFR1). Interaction of TNF $\alpha$ with trimerized TNFR1 results in the formation of Complex I. This short-lived membranesignalling complex is indeed 'complex', and is composed of the adaptor molecule TNF receptor-associated death domain (TRADD), TNF receptor-associated factor 2 (TRAF2), RIPK1, cellular inhibitor of apoptosis 1 (cIAP1), cIAP2, and the linear ubiquitin chain assembly complex (LUBAC). Complex I appears to be a critical checkpoint for cell death versus cell survival signalling, as engagement with TNFR1 can also lead to NFkB activation and pro-inflammatory cytokine release rather than cell death. How do cells commit to survival or death following TNF $\alpha$ ligation? Separation of responses involves phosphorylation events, but also the ubiquination state of several key Complex I components by LUBAC, IAPs and cylindromatosis (CYLD). The ubiquination state of molecules allows for recruitment or activation of the inhibitor of $\mathrm{KB}$ kinase (IKK) complex (which includes NEMO) to control nuclear translocation of NF- $\mathrm{KB}$ dimers, or for cell death, as in the case of RIPK1. On deubiquitination of the K63 and linear ubiquitin chains of RIPK1 by deubiquitinases, RIPK1 loses its 


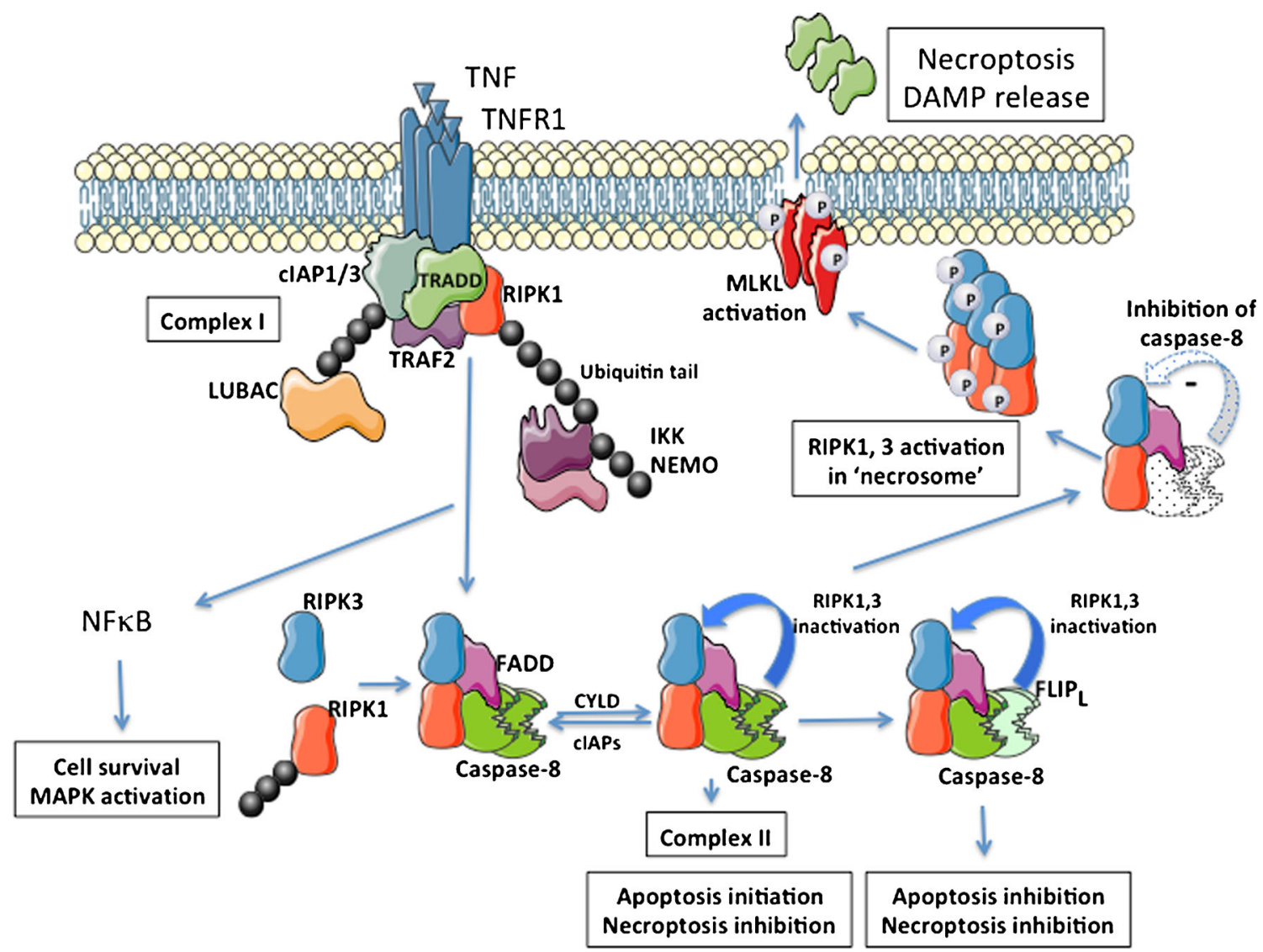

Fig. 2 Necroptosis can be triggered through TNF receptor 1 (TNFR1), as well as other death receptors in the TNF superfamily, Toll-like receptor 3 (TLR3) and TLR4. Interaction of TNF with trimerized TNFR1 results in the formation of Complex I at the membrane. Activation of the inhibitor of $\kappa \mathrm{B}$ kinase (IKK) complex (which includes NEMO) controls nuclear translocation of NF- $\mathrm{KB}$ dimers, leading to survival. In contrast, on deubiquitination of RIPK1, it loses its pro-survival function and promotes cell death by associating with RIP3. Complex 1 dissociates from the membrane into a cytosolic complex with recruitment of FADD and caspase- 8 to form Complex II. The apoptotic caspase cascade can be

default pro-survival function and promotes cell death by associating with RIP3. Interestingly, patients with mutations in the E3 ligase subunit of LUBAC exhibit chronic inflammation, increased cytokine expression in response to IL- $1 \beta$, cardiomyopathy, and susceptibility to pyogenic bacteria due to impaired NF- $\mathrm{KB}$ activation [80]. cIAPs are ubiquitin E3 ligases that are required for induction of NF-kB by TNF $\alpha$. Smac mimetics (SMs) trigger autoubiquitination and degradation of IAPs, and the subsequent depletion of cIAP1, cIAP2, and XIAP biases cell responses to death. Importantly IAPs, along with caspase- 8 and cFLIP participate to keep the molecules RIPK1 and RIPK3 in check to prevent unintended apoptosis, or necroptosis and inflammation.

Following surface ligand engagement, Complex 1 can dissociate from the membrane into a cytosolic complex with recruitment of FADD and caspase-8. This has been referred to as Complex IIa. While dimerization of caspase- 8 in this setting leads to apoptosis via auto-processing of caspase- 8 blocked by heterodimerization of caspase- 8 with cFLIP. If levels of cFLIP-L are sufficient, Complex 2 does not have any killing activity. Both caspase- 8 dimers and caspase-8/cFLIP heterodimers can cleave and inactivate the essential necroptosis mediators RIPK1 and RIPK3. cIAPs can be disabled by SMAC mimetics and can increase RIPK1 to levels high enough to auto-activate the complex and initiate necroptosis. Functional RIPK1 and RIPK3 interact via RHIM motifs and recruit mixed lineage kinase domain-like (pMLKL) protein, which translocates to the membrane to activate rupture and release of cytosolic DAMPs

and downstream caspase-3 activation, the apoptotic caspase cascade can be blocked by heterodimerization of caspase- 8 with cFLIP, an enzyme-inactive homolog of caspase-8. If cFLIP is absent or low, active caspase-8 induces apoptosis by promoting caspase-3 and/or Bid cleavage. Furthermore, both caspase- 8 dimers and caspase-8/cFLIP heterodimers can cleave and inactivate the essential necroptosis mediators RIPK1, RIPK3, and cylindromatosis (CYLD) to prevent necroptosis. Thus apoptosis and necroptosis are counter balanced forms of cell death, and caspase- 8 activity directs the cellular fate. Indeed, it has been recently appreciated that a key function of caspase- 8 may be to prevent necroptosis rather than allow apoptosis per se $[55,81,82]$. The genetic loss of caspase- 8 results in embryonic death in mice, which can be rescued by concurrent genetic deletion of RIPK3 [81]. Thus, in some cell types, inhibition of caspase- 8 (or its upstream adaptor FADD) may block apoptosis, but trigger necroptosis. Functional RIPK1 and RIPK3 are allowed to interact via RIP 
homotypic interaction motif (RHIM), which results in amyloid-like complexes essential for recruitment and activation of the mixed lineage kinase domain-like (pMLKL) protein. Interaction of RIPK1 and RIPK3 leads to mutual phosphorylation and activation. Subsequent phosphorylation of the C-terminal pseudokinase domain of MLKL by RIPK3 induces conformational changes that allow pMLKL translocation to membranes. pMLKL was initially identified as a target of a small molecule inhibitor (necrosulfonamide) in screening for necroptosis inhibitors [83-85]. It remains unclear how pMLKL precisely results in the terminal event of plasmamembrane rupture, but this raises another potential therapeutic intervention through the use of necrosulfonamides. It is important to note that while TNF $\alpha$, in combination with a pancaspase inhibitor, is sufficient to activate RIPK3-dependent necroptosis in many cell types, other cells additionally require an cIAP antagonist for robust killing, and yet others remain resistant $[54,86]$. This might confound conclusions regarding the role of necroptosis in various organ transplant models, as the cellular composition of organs is obviously complex. Furthermore, T lymphocytes undergo RIPK1- and RIPK3dependent necroptosis when FADD or caspase- 8 function is compromised, but death is induced only after stimulation of the TCR. The mechanism by which TCR signalling is linked to RIPK1 and RIPK3 for necroptosis is unclear, but the recipient immune system is likely to be affected by 'death modifying' therapeutics given systemically [87].

\section{Necroptosis in Ischemia/Reperfusion Injury and Transplant Models}

While necrosis of kidney has been demonstrated in I/R injury in animal models, the identification of necroptosis by its response to Nec-1 inhibition has been complicated by recently noted 'off target' effects $[69,88]$. We have recently demonstrated that isolated murine tubular epithelial cells (TEC), grown as monolayers in vitro, are capable of enhanced death when treated with both TNF $\alpha$ and caspase inhibition, with release of HMGB1 and inhibition by Nec-1, in keeping with a regulated necrosis form of cell death [89]. The inability to trigger necrosis in TEC lacking RIPK3 provides more substantial support for their ability to undergo necroptosis. Furthermore, the loss of kidney RIPK3 by genetic deletion ameliorated acute kidney $\mathrm{I} / \mathrm{R}$ injury, similar to that observed with RIPK1 inhibition by Nec-1 [89-91]. Thus, necroptosis appears to be a mechanism for inflammatory kidney injury. Necroptosis was shown to be a major mechanism of proximal tubular cell death in cisplatin-induced nephrotoxic AKI in which cell death of primary culture proximal tubular cells was clearly RIPK1 and RIPK3 dependent [92]. In a contrastinduced acute kidney injury (CIAKI) model, Nec-1 prevented osmotic nephrosis and injury, but also prevented contrast- induced dilation of peritubular capillaries, suggesting a novel role for the RIP1 kinase domain in regulating microvascular hemodynamics [93]. Thus, while we suggest that the primary benefit observed in these models was by the elimination of necroptosis, potential effects of RIPK 3 on kidney vasculature homeostasis and AKI have not been excluded. In distinct contrast to these reports, protection in kidney I/R injury, as well as reduced injury of isolated tubules, was demonstrated using ferrostatin, suggesting that ferroptosis is also involved in $I / R$ injury [75]. Divergent results in organ I/R injury models suggest that regulated necrosis within organs may vary with the inflammatory challenge, differences in cell types, culture conditions, in vivo vs. in vitro model systems, and reflects the complexity of cell types involved in each organ. Such differences may need to be resolved prior to extensive clinical testing, although therapeutic agents that block several forms of regulated necrosis may remain useful. Nec-1 may attenuate non-necroptosis forms of regulated necrosis, but newer analogues may address this issue of specificity $[94,95]$. As noted, a current challenge in assessing regulated necrosis in vivo has been the lack of reproducible and quantifiable methods to measure tissue necrosis. The use of perfused ethidium homodimer, which occurs almost exclusively with loss of cell membrane integrity in necrosis [96], aids in quantifying necrosis in vivo, but does not differentiate the various forms of necrosis. The lack of specific markers to distinguish various forms of regulated necrosis has also hampered our analyses. Antibodies that detect human phosphorylated MLKL were recently reported to detect necrotic hepatocytes, and if validated in other models, may be useful in transplant samples [97].

Regulated necrosis has been demonstrated in many cell types and organs. Nec-1 was effective in alleviating hypoxiaischemia-induced oxidative brain injury [98]. Nec-1 also had benefit in inhibiting myocardial cell death and infarct size, via a mechanism independent of the MPTP [99]. In a mouse model in which caspase-8 was deleted in liver by using cre/loxP conditional knockouts, liver histology under basal conditions had inflammatory infiltrates of neutrophils and monocytes and increased serum transaminases. This suggests that loss of caspase-8 alone in hepatocytes promotes liver inflammation. Under inflammatory stress, caspase- 8 deletion protected mice from apoptosis liver injury (using anti Fas antibody) or lipopolysaccharides (LPS), but increased necrotic damage and reduced survival times of mice given concanavalin A. Furthermore, concurrent disruption of both nuclear factor- kB (NFkB) essential modulator (NEMO) as well as Caspase-8 resulted in massive liver necrosis, cholestasis, and biliary lesions. [100]. Necrosis rather than apoptosis may be the major form of cell death found in lungs prior to transplant, and be related to duration of hypothermic preservation [101, 102]. Thus, in most organs, it appears that there is a balance between apoptosis and necroptosis, but generally any disruption of the control of necroptosis leads to injury, which may be attenuated by agents. 
What is the relative contribution of necrosis versus apoptosis to overall donor organ dysfunction with $\mathrm{I} / \mathrm{R}$ injury? Clearly, the degree of parenchymal cells loss from either apoptosis or necrosis directs the apparent organ (and in particular kidney) dysfunction in short-term $\mathrm{I} / \mathrm{R}$ injury models. Once a threshold degree of parenchymal cell loss exceeds the ability of the organ to function, the original mechanism of death becomes somewhat irrelevant. With necrosis, the release of DAMP, including HMGB1, could have a direct effect on renal tubular cells. Following kidney I/R injury, increased expression of TLR4 on TEC in the first 5-10 days coincides with the expression and presumably availability of HMGB1 [103, 104]. This may augment or prolong TEC injury, as TLR4 signalling can trigger necroptosis, and loss of TLR4 on kidney cells is protective in I/R injury. HMGB1 may also contribute to the activation of infiltrating effector cells, and in particular, NK cells $[105,106]$. There is an intriguing recent report using a rat renal allograft model in which AKI caused distant pulmonary injury following engraftment. PARP-1 expression (reflecting parthanatos form of regulated necrosis), as well RIPK1 and RIPK3, was significantly enhanced in injured lungs. Importantly, injury was reduced after treatment with Nec-1 or/and the PARP-1 inhibitor 3-aminobenzamide (3-AB) [107••]. As apoptosis can also have distant effects on organs, it may be that we need to consider therapeutic strategies that target multiple forms of cell death, including apoptosis and necroptosis, as been shown in brain I/R injury models [108] and myocardial infarct studies [109] and now PARP-1, to limit transplant organ injury as well as distant 'cross talk' injury.

\section{Necroptosis in Solid Organ Transplants}

Consistent with regulation of necroptosis by caspase- 8 and in vitro results using cultured TEC, we observed increased injury and necrosis in donor kidneys with shRNA caspase-8 silencing following transplantation into fully allogeneic recipients without immunosuppression [49•, 89]. These results are consistent with the loss of necroptosis regulation by caspase8 , and promotion of allogeneic responses by subsequent inflammation. Indeed, inhibition of downstream caspases (i.e. caspase-3) may not have had a similar effect. Alternatively, there may also have been a loss of anti-inflammatory / tolerogenic membrane bound vesicles generated by apoptotic cells [110-114]. We have also noted that the regulation of cell death, including necroptosis by caspase- 8 , is $\mathrm{pH}$ sensitive, which may have accounted for differences in blocking caspase- 8 in short-term I/R injury compared to long-term transplant models. In direct support of necroptosis contributing to kidney allograft injury, elimination of RIPK3 in donor kidneys promoted long-term allograft survival without immunosuppression. Importantly, the elimination of necroptosis and reduction of DAMP release, such as of HMGB1 posttransplant, did not result in tolerance per se. While $\mathrm{CD}^{+}$ cellular infiltration and tubulitis were reduced in RIPK3-/- as compared to wild type kidneys, tubulitis and other features of rejection remained evident in grafts of RIPK3-/- longterm survivors. Thus, it appears that the benefit of RIPK3-/ - deletion is related to attenuation but not elimination of alloimmune responses. It is tempting to speculate that the threshold for tolerance induction may be lowered in kidney transplants with elimination of necroptosis and subsequent inflammation. In relation to this, kidneys have an endogenous capacity to downregulate immune responses post transplant. Mouse kidney transplants have high spontaneous acceptance rates of $20-30 \%$, which is not seen in heart transplants using the same strain combinations [115-117]. While beyond of the scope of this review, the high expression of FasL, SPI-6, TNF $\alpha$, TGF $\beta$, IL-15, IL-37, osteopontin, PDL2, erythropoietin (EPO) and other immunoregulatory or cytoprotective molecules within the kidney may have augmented the capacity of transplanted kidneys to downregulate rejection responses in the absence of RIPK3 mediated necroptosis [30••, 115, 118-121].

In contrast, RIPK3 deficiency in donor heart grafts induced long-term graft survival compared with wild type donor grafts, but recipients required a 'sub-threshold' immunosuppression with sirolimus to achieve this. While observed differences in survival of RIPK3-/- kidneys compared to heart transplants remain speculative, loss of RIPK3-mediated necroptosis alone in donor hearts was insufficient to promote long-term survival. Similar to kidney transplants, lymphocyte infiltration and vascular damage were reduced but not eliminated in RIPK $3^{-/}$heart grafts. There has been speculation as to the capacity of myocytes and endothelial cells to undergo necroptosis, and indeed whether the benefit observed using RIPK3-/- or Nec-1 administration in I/R injury or transplant studies was related to loss of necroptosis in the endothelial or other parenchymal compartment. Conceptually, this is difficult to address, as all parenchymal cells depend on endothelial cell homeostasis and an adequate blood supply. We did not test the capacity of isolated cardiomyocytes to undergo necroptosis, although earlier reports suggest that this occurs in ischemic models [109]. In vitro studies using flow cytometry and release of HMGB1 demonstrated in our hands that micro vascular endothelial cells (MVEC) can undergo RIK3 mediated necroptosis, and thus, this may be a therapeutic target in clinical transplantation.

An outstanding question remains as to what is the direct contribution of necroptosis to alloimmune activation, and whether necroptosis is the 'chicken or egg' in inflammation. While there is evidence for immune recognition of necrotic cells, there is a surprising paucity of unequivocal evidence that necrosis per se alters associated alloimmune responses. While apoptosis is generally considered to be non- 
immunogenic, apoptotic cell death from tissue injury rather than developmental programs such as thymocyte deletion should conceptually trigger host defense pathways. Several reports have suggested this is indeed the case and that under some conditions, apoptosis is pro-inflammatory. In contrast, necrosis by its release of non-membrane enclosed cytosolic contents (CDAMPs) is generally assumed to always be proinflammatory. Necroptosis, with its similarities to other forms of necrosis, is unlikely to display different properties in regulating inflammation but this too has not been established. Interestingly, organ-specific knockouts of HMGB1 did not lessen, but rather worsened LPS-induced or injury-induced inflammation, likely related to its key role in maintaining genome homeostasis and cell survival. Thus definitive proof on this point will be difficult experimentally, as many other CDAMPs have similarly important cellular roles $[60 \bullet \cdot$. Necroptotic material can be co-ingested in vitro by endocytosis-macropinocytosis, and coincubation of either apoptotic or necrotic cells with macrophages did not result in differences in cytokine induction [122]. Similarly, B16 mouse melanoma cells, in which necroptosis was triggered by inducible expression of the FADD-death domain, were unable to induce maturation of dendritic cells (DCs) [123]. In a skin transplant model, necrotic cells from skeletal muscle tissue injected subcutaneously into naive C57BL/6 mice with and without alloantigen resulted in vigorous alloresponses, but there was no differential $\mathrm{T}$ cell activation/maturation associated with necrosis, and skin graft survival was not altered [124]. As regulated necrosis is likely related to host defense, it is reasonable to consider necroptosis and infection. One of the continuing challenges in transplantation is balancing immunosuppression adequately to prevent rejection, yet allowing resistance to opportunistic pathogens, particularly viruses. It is of great interest that regulated necrosis can occur not only following $\mathrm{I} / \mathrm{R}$ injury and perhaps with rejection, but also as a result of viral infection. Given the fact that necroptosis represents a 'failsafe' mechanism for host defense from viruses, it is not surprising that viruses that encode caspase- 8 inhibitors are susceptible to host cell necroptosis. Murine CMV encodes several viral cell death inhibitors, one of which is the viral inhibitor of caspase-8-induced apoptosis (vICA). However, the virus also encodes M45/vIRA is a RHIM-containing viral cell death inhibitor that binds to RIPK3, and infected cells are spared from necroptosis. Similar effects have recently been noted with human CMV [125-127]. It remains to be tested if certain viruses promote rejection or prevent tolerance through effects on cell death and regulated necrosis. At present, it is not entirely clear how products of necrosis promote inflammation and divergent results exist. Surgical procedures that cause tissue injury and release of DAMP can promote alloantibody expression in sensitized transplant recipients [128]. In transplants, loss of RIPK3 can reduce DAMP expression and attenuate rejection, but not induce tolerance. This would support that there is a link between necrosis, innate inflammation and alloimmunity. Studies that identify the mechanisms by which innate inflammation might drive alloimmunity will benefit from the increasing availability of novel models with genetic deletions, as well as new pharmacologic agents.

\section{Conclusions}

In summary, the previous partitioning of cell death simply into apoptosis and necrosis was obviously naïve and is now somewhat historic. From basic discovery research in diverse areas of science including developmental biology, immunology, cancer, tissue injury and most recently transplantation, our appreciation of diverse forms of regulated cell death in clinical conditions has evolved considerably. Both apoptosis and necroptosis are directly relevant to organ injury, and importantly, may be amenable by targeting in transplantation using new classes of drugs such as necrostatins. A challenge remains in understanding the counter-balanced nature of these pathways and how perturbation of one pathway might trigger others. Additionally, it is not clear in clinical scenarios whether targeting of regulated death will require multiple agents, or whether control will be best delivered to organs prior to transplantation or delivered to the recipient in the immediate post transplant period. Nonetheless, it is exciting in transplantation when paradigms change and new options emerge to improve outcomes. Identifying new forms of regulated cell death will no doubt provide novel therapeutic options that will reduce organ inflammation. It remains to be seen whether this will translate to improved long-term survival.

Acknowledgments MJH is the holder of the Shire Chair in Nephrology, Transplantation and Renal Regeneration of Université de Montréal. We thank the J.-L. Lévesque Foundation for renewed support. This work was supported by research grants from the Canadian Institutes of Health Research (CIHR) (for MJH, MOP-15447, MOP-89869 and for AMJ, MOP-115048, MOP-111180). MJH and AJ are members of the Canadian National Transplant Research Program.

\section{Compliance with Ethics Guidelines}

Conflict of Interest Marie-Josee Hebert and Anthony M. Jevnikar declare that they have no conflict of interest.

Human and Animal Rights and Informed Consent This article does not contain any studies with human subjects performed by any of the authors. All procedures performed in studies involving animals were in accordance with the ethical standards of the institution or practice at which the studies were conducted. 


\section{References}

Papers of particular interest, published recently, have been highlighted as:

- Of importance

•- Of major importance

1. Kerr JF, Wyllie AH, Currie AR. Apoptosis: a basic biological phenomenon with wide-ranging implications in tissue kinetics. Br J Cancer. 1972;26(4):239-57.

2. Galluzzi L, Vitale I, Abrams JM, et al. Molecular definitions of cell death subroutines: recommendations of the nomenclature committee on cell death 2012. Cell Death Differ. 2012;19(1): $107-20$.

3. Zheng X, Zhang X, Sun H, et al. Protection of renal ischemia injury using combination gene silencing of complement 3 and caspase 3 genes. Transplantation. 2006;82(12):1781-6.

4. Yang C, Zhao T, Zhao Z, et al. Serum-stabilized naked caspase-3 siRNA protects autotransplant kidneys in a porcine model. Mol Ther. 2014;22(10):1817-28.

5. Zhang $\mathrm{X}$, Zheng $\mathrm{X}$, Sun $\mathrm{H}$, et al. Prevention of renal ischemic injury by silencing the expression of renal caspase 3 and caspase 8. Transplantation. 2006;82(12):1728-32.

6. Zhang YX, Fan H, Shi Y, et al. Prevention of lung ischemiareperfusion injury by short hairpin RNA-mediated caspase-3 gene silencing. J Thorac Cardiovasc Surg. 2010;139(3):758-64.

7. Mueller TH, Kienle K, Beham A, Geissler EK, Jauch KW, Rentsch M. Caspase 3 inhibition improves survival and reduces early graft injury after ischemia and reperfusion in rat liver transplantation. Transplantation. 2004;78(9):1267-73.

8. Balsam LB, Mokhtari GK, Jones S, et al. Early inhibition of caspase-3 activity lessens the development of graft coronary artery disease. J Heart Lung Transplant. 2005;24(7):827-32.

9. Tanaka M, Nakae S, Terry RD, et al. Cardiomyocyte-specific Bcl2 overexpression attenuates ischemia-reperfusion injury, immune response during acute rejection, and graft coronary artery disease. Blood. 2004;104(12):3789-96.

10. Kown MH, Miniati DN, Jahncke CL, et al. Bcl-2-mediated inhibition of apoptosis in rat cardiac allografts worsens development of graft coronary artery disease. J Heart Lung Transplant. 2003;22(9):986-92.

11. El-Gibaly AM, Scheuer C, Menger MD, Vollmar B. Improvement of rat liver graft quality by pifithrin-alpha-mediated inhibition of hepatocyte necrapoptosis. Hepatology. 2004;39(6):1553-62.

12. Baskin-Bey ES, Washburn K, Feng S, et al. Clinical trial of the Pan-caspase inhibitor, IDN-6556, in human liver preservation injury. Am J Transplant. 2007;7(1):218-25.

13. Schuler M, Bossy-Wetzel E, Goldstein JC, Fitzgerald P, Green DR. p53 induces apoptosis by caspase activation through mitochondrial cytochrome c release. J Biol Chem. 2000;275(10): 7337-42.

14. Wolff S, Erster S, Palacios G, Moll UM. p53's mitochondrial translocation and MOMP action is independent of Puma and Bax and severely disrupts mitochondrial membrane integrity. Cell Res. 2008;18(7):733-44.

15. Molitoris BA, Dagher PC, Sandoval RM, et al. siRNA targeted to p53 attenuates ischemic and cisplatin-induced acute kidney injury. J Am Soc Nephrol. 2009;20(8):1754-64.

16. Imamura R, Isaka Y, Sandoval RM, et al. Intravital two-photon microscopy assessment of renal protection efficacy of siRNA for p53 in experimental rat kidney transplantation models. Cell Transplant. 2010;19(12):1659-70.

17. Kelly KJ, Plotkin Z, Vulgamott SL, Dagher PC. P53 mediates the apoptotic response to GTP depletion after renal ischemia- reperfusion: protective role of a p53 inhibitor. J Am Soc Nephrol. 2003;14(1):128-38.

18. Dagher PC, Mai EM, Hato T, et al. The p53 inhibitor pifithrinalpha can stimulate fibrosis in a rat model of ischemic acute kidney injury. Am J Physiol Renal Physiol. 2012;302(2):F284-91.

19. Hoshino A, Matoba S, Iwai-Kanai E, et al. p53-TIGAR axis attenuates mitophagy to exacerbate cardiac damage after ischemia. $\mathrm{J}$ Mol Cell Cardiol. 2012;52(1):175-84.

20. Liu P, Xu B, Cavalieri TA, Hock CE. Inhibition of p53 by pifithrin-alpha reduces myocyte apoptosis and leukocyte transmigration in aged rat hearts following 24 hours of reperfusion. Shock. 2008;30(5):545-51.

21. Sutton TA, Hato T, Mai E, et al. p53 is renoprotective after ischemic kidney injury by reducing inflammation. J Am Soc Nephrol. 2013;24(1):113-24.

22. Barry M, Heibein JA, Pinkoski MJ, et al. Granzyme B shortcircuits the need for caspase 8 activity during granule-mediated cytotoxic T-lymphocyte killing by directly cleaving Bid. Mol Cell Biol. 2000;20(11):3781-94.

23. Metkar SS, Wang B, Ebbs ML, et al. Granzyme B activates procaspase-3 which signals a mitochondrial amplification loop for maximal apoptosis. J Cell Biol. 2003;160(6):875-85.

24. Ewen CL, Kane KP, Bleackley RC. Granzyme H induces cell death primarily via a Bcl-2-sensitive mitochondrial cell death pathway that does not require direct Bid activation. Mol Immunol. 2013;54(3-4):309-18.

25. Catalan E, Jaime-Sanchez P, Aguilo N, Simon MM, Froelich CJ, Pardo J. Mouse cytotoxic T cell-derived granzyme B activates the mitochondrial cell death pathway in a Bim-dependent fashion. J Biol Chem. 2015;290(11):6868-77.

26. Muthukumar T, Dadhania D, Ding R, et al. Messenger RNA for FOXP3 in the urine of renal-allograft recipients. New Eng J Med. 2005;353(22):2342-51.

27. Sutton VR, Estella E, Li C, et al. A critical role for granzyme B, in addition to perforin and TNFalpha, in alloreactive CTL-induced mouse pancreatic beta cell death. Transplantation. 2006;81(2): $146-54$.

28. Sleater M, Diamond AS, Gill RG. Islet allograft rejection by contact-dependent CD8+ T cells: perforin and FasL play alternate but obligatory roles. Am J Transplant. 2007;7(8):1927-33.

29. Gomez L, Raisky O, Chalabreysse L, Verschelde C, BonnefoyBerard N, Ovize M. Link between immune cell infiltration and mitochondria-induced cardiomyocyte death during acute cardiac graft rejection. Am J Transplant. 2006;6(3):487-95.

30.• Lau A, Khan K, Pavlosky A, et al. Serine protease inhibitor-6 inhibits granzyme B-mediated injury of renal tubular cells and promotes renal allograft survival. Transplantation. 2014;98(4): 402-10. This is the first report of the role of RIPK3 mediated necroptosis in solid organ transplantation.

31. Du C, Wang S, Diao H, Guan Q, Zhong R, Jevnikar AM. Increasing resistance of tubular epithelial cells to apoptosis by shRNA therapy ameliorates renal ischemia-reperfusion injury. Am J Transplant. 2006;6(10):2256-67.

32. Kayser D, Einecke G, Famulski KS, et al. Donor Fas is not necessary for T-cell-mediated rejection of mouse kidney allografts. Am J Transplant. 2008;8(10):2049-55.

33. Du C, Jiang J, Guan Q, et al. Renal tubular epithelial cell selfinjury through $\mathrm{Fas} / \mathrm{Fas}$ ligand interaction promotes renal allograft injury. Am J Transplant. 2004;4(10):1583-94.

34. Linkermann A, Himmerkus N, Rolver L, et al. Renal tubular Fas ligand mediates fratricide in cisplatin-induced acute kidney failure. Kidney Int. 2011;79(2):169-78.

35. Grazia TJ, Plenter RJ, Weber SM, et al. Acute cardiac allograft rejection by directly cytotoxic CD4 T cells: parallel requirements for Fas and perforin. Transplantation. 2010;89(1):33-9. 
36. Askenasy N, Yolcu ES, Wang Z, Shirwan H. Display of Fas ligand protein on cardiac vasculature as a novel means of regulating allograft rejection. Circulation. 2003;107(11): 1525-31.

37. Plenter RJ, Grazia TJ, Nelson DP, Zamora MR, Gill RG, Pietra BA. Ectopic expression of Fas Ligand on cardiomyocytes renders cardiac allografts resistant to CD4(+) T-cell mediated rejection. Cell Immunol. 2015;293(1):30-3.

38. White LE, Cui Y, Shelak CM, Lie ML, Hassoun HT. Lung endothelial cell apoptosis during ischemic acute kidney injury. Shock. 2012;38(3):320-7. This work highlights the importance of acute kidney injury as a major trigger for distant organ microvascular damage.

39. Hassoun HT, Lie ML, Grigoryev DN, Liu M, Tuder RM, Rabb H. Kidney ischemia-reperfusion injury induces caspase-dependent pulmonary apoptosis. Am J Physiol Renal Physiol. 2009;297(1): F125-37.

40. An S, Hishikawa Y, Liu J, Koji T. Lung injury after ischemiareperfusion of small intestine in rats involves apoptosis of type II alveolar epithelial cells mediated by TNF-alpha and activation of Bid pathway. Apoptosis. 2007;12(11):1989-2001.

41. Zhao H, Ning J, Lemaire A, et al. Necroptosis and parthanatos are involved in remote lung injury after receiving ischemic renal allografts in rats. Kidney Int. 2015;87(4):738-48.

42. Sirois I, Raymond MA, Brassard N, et al. Caspase-3-dependent export of TCTP: a novel pathway for antiapoptotic intercellular communication. Cell Death Differ. 2011;18(3):549-62. This paper demonstrates that caspase-3 activation during apoptosis triggers the release of membrane vesicles that differ from classical apoptotic bodies.

43. Sirois I, Groleau J, Pallet N, et al. Caspase activation regulates the extracellular export of autophagic vacuoles. Autophagy. 2012;8(6):927-37.

44. Loyer X, Vion AC, Tedgui A, Boulanger CM. Microvesicles as cell-cell messengers in cardiovascular diseases. Circ Res. 2014;114(2):345-53.

45. Levine SJ. Molecular mechanisms of soluble cytokine receptor generation. J Biol Chem. 2008;283(21):14177-81.

46.• Gao B, Moore C, Porcheray F, et al. Pretransplant IgG reactivity to apoptotic cells correlates with late kidney allograft loss. Am J Transplant. 2014;14(7):1581-91. This paper describes the importance of humoral reactivity to apoptotic cells on long-term renal allograft survival.

47. Porcheray F, Fraser JW, Gao B, et al. Polyreactive antibodies developing amidst humoral rejection of human kidney grafts bind apoptotic cells and activate complement. Am J Transplant. 2013;13(10):2590-600.

48.• Cardinal H, Dieude M, Brassard N, et al. Antiperlecan antibodies are novel accelerators of immune-mediated vascular injury. Am J Transplant. 2013;13(4):861-74. This paper characterizes autoantidobies to a perlecan epitope released by apoptotic endothelial cells as a novel accelerator of vascular injury in renal transplantation.

49. Linkermann A, Hackl MJ, Kunzendorf U, Walczak H, Krautwald S, Jevnikar AM. Necroptosis in immunity and ischemiareperfusion injury. Am J Transplant. 2013;13(11):2797-804. This reviews the current understanding of regulated forms of necrosis in transplantation.

50. Cavaille-Coll M, Bala S, Velidedeoglu E, et al. Summary of FDA workshop on ischemia reperfusion injury in kidney transplantation. Am J Transplant. 2013;13(5):1134-48.

51. Garg AD, Nowis D, Golab J, Vandenabeele P, Krysko DV, Agostinis P. Immunogenic cell death, DAMPs and anticancer therapeutics: an emerging amalgamation. Biochim Biophys Acta. 2010;1805(1):53-71.
52. Krysko DV, Agostinis P, Krysko O, et al. Emerging role of damage-associated molecular patterns derived from mitochondria in inflammation. Trends Immunol. 2011;32(4):157-64.

53. Chong AS, Alegre ML. Transplantation tolerance and its outcome during infections and inflammation. Immunol Rev. 2014;258(1): 80-101.

54. Newton K. RIPK1 and RIPK3: critical regulators of inflammation and cell death. Trends Cell Biol. 2015.

55. Linkermann A, Green DR. Necroptosis. New Eng J Med. 2014;370(5):455-65.

56. Chan FK, Luz NF, Moriwaki K. Programmed necrosis in the cross talk of cell death and inflammation. Ann Rev Immunol. 2014.

57. Kaczmarek A, Vandenabeele P, Krysko DV. Necroptosis: the release of damage-associated molecular patterns and its physiological relevance. Immunity. 2013;38(2):209-23.

58. Kroemer G, Galluzzi L, Vandenabeele P, et al. Classification of cell death: recommendations of the nomenclature committee on cell death 2009. Cell Death Differ. 2009;16(1):3-11.

59. Vercammen D, Beyaert R, Denecker G, et al. Inhibition of caspases increases the sensitivity of L929 cells to necrosis mediated by tumor necrosis factor. J Exp Med. 1998;187(9):1477-85.

60.• Pasparakis M, Vandenabeele P. Necroptosis and its role in inflammation. Nature. 2015;517(7534):311-20. This is a definitive and exhaustive review of the mechanisms and forms of regulated necrosis.

61. Scaffidi P, Misteli T, Bianchi ME. Release of chromatin protein HMGB 1 by necrotic cells triggers inflammation. Nature. 2002;418(6894):191-5.

62. Beyer C, Stearns NA, Giessl A, Distler JH, Schett G, Pisetsky D. The extracellular release of DNA and HMGB1 from Jurkat T cells during in vitro necrotic cell death. Innate Immun. 2012.

63. Andrade-Oliveira V, Campos EF, Goncalves-Primo A, et al. TLR4 mRNA levels as tools to estimate risk for early posttransplantation kidney graft dysfunction. Transplantation. 2012;94(6):589-95.

64. Goldstein DR, Tesar BM, Akira S, Lakkis FG. Critical role of the Toll-like receptor signal adaptor protein MyD88 in acute allograft rejection. J Clin Invest. 2003;111(10):1571-8.

65. Liu G, Zhao Y. Toll-like receptors and immune regulation: their direct and indirect modulation on regulatory CD4+CD25+ T cells. Immunology. 2007;122(2):149-56.

66. Vilaysane A, Chun J, Seamone ME, et al. The NLRP3 inflammasome promotes renal inflammation and contributes to CKD. J Am Soc Nephrol. 2010;21(10):1732-44.

67. Sawai H, Domae N. Discrimination between primary necrosis and apoptosis by necrostatin-1 in Annexin V-positive/propidium iodide-negative cells. Biochem Biophys Res Commun. 2011;411(3):569-73.

68. Gunther C, Martini E, Wittkopf N, et al. Caspase-8 regulates TNFalpha-induced epithelial necroptosis and terminal ileitis. Nature. 2011;477(7364):335-9.

69. Vanden Berghe T, Linkermann A, Jouan-Lanhouet S, Walczak H, Vandenabeele P. Regulated necrosis: the expanding network of non-apoptotic cell death pathways. Nat Rev Mol Cell Biol. 2014;15(2):135-47.

70. Sosna J, Voigt S, Mathieu S, et al. TNF-induced necroptosis and PARP-1-mediated necrosis represent distinct routes to programmed necrotic cell death. Cell Mol Life Sci. 2014;71(2): 331-48.

71. Tait SW, Oberst A, Quarato G, et al. Widespread mitochondrial depletion via mitophagy does not compromise necroptosis. Cell Rep. 2013;5(4):878-85.

72. Lawlor KE, Khan N, Mildenhall A, et al. RIPK3 promotes cell death and NLRP3 inflammasome activation in the absence of MLKL. Nat Commun. 2015;6:6282.

73. Anders HJ, Muruve DA. The inflammasomes in kidney disease. J Am Soc Nephrol. 2011;22(6):1007-18. 
74. Friedmann Angeli JP, Schneider M, Proneth B, et al. Inactivation of the ferroptosis regulator Gpx4 triggers acute renal failure in mice. Nat Cell Biol. 2014;16(12):1180-91.

75. Linkermann A, Skouta R, Himmerkus N, et al. Synchronized renal tubular cell death involves ferroptosis. Proc Natl Acad Sci U S A. 2014;111(47):16836-41.

76. Dillon CP, Weinlich R, Rodriguez DA, et al. RIPK1 blocks early postnatal lethality mediated by caspase- 8 and RIPK3. Cell. 2014;157(5):1189-202.

77. Tait SW, Green DR. Caspase-independent cell death: leaving the set without the final cut. Oncogene. 2008;27(50):6452-61.

78. Tait SW, Ichim G, Green DR. Die another way-non-apoptotic mechanisms of cell death. J Cell Sci. 2014;127(Pt 10):2135-44.

79. Wilson NS, Dixit V, Ashkenazi A. Death receptor signal transducers: nodes of coordination in immune signaling networks. Nat Immunol. 2009;10(4):348-55.

80. Boisson B, Laplantine E, Prando C, et al. Immunodeficiency, autoinflammation and amylopectinosis in humans with inherited HOIL-1 and LUBAC deficiency. Nat Immunol. 2012;13(12): 1178-86.

81. Oberst A, Dillon CP, Weinlich R, et al. Catalytic activity of the caspase-8-FLIP(L) complex inhibits RIPK3-dependent necrosis. Nature. 2011;471(7338):363-7.

82. Green DR, Oberst A, Dillon CP, Weinlich R, Salvesen GS. RIPKdependent necrosis and its regulation by caspases: a mystery in five acts. Mol Cell. 2011;44(1):9-16.

83. Green DR. Pseudokiller, qu'est-ce que c'est? Immunity. 2013;39(3):421-2.

84. Zhao J, Jitkaew S, Cai Z, et al. Mixed lineage kinase domain-like is a key receptor interacting protein 3 downstream component of TNF-induced necrosis. Proc Natl Acad Sci U S A. 2012;109(14): $5322-7$

85. Sun L, Wang H, Wang Z, et al. Mixed lineage kinase domain-like protein mediates necrosis signaling downstream of RIP3 kinase. Cell. 2012;148(1-2):213-27.

86. Laukens B, Jennewein C, Schenk B, et al. Smac mimetic bypasses apoptosis resistance in FADD- or caspase-8-deficient cells by priming for tumor necrosis factor alpha-induced necroptosis. Neoplasia. 2011;13(10):971-9.

87. Lu JV, Weist BM, van Raam BJ, et al. Complementary roles of Fas-associated death domain (FADD) and receptor interacting protein kinase-3 (RIPK3) in T-cell homeostasis and antiviral immunity. Proc Natl Acad Sci U S A. 2011;108(37):15312-7.

88. Linkermann A, Brasen JH, Darding M, et al. Two independent pathways of regulated necrosis mediate ischemia-reperfusion injury. Proc Natl Acad Sci U S A. 2013;110(29):12024-9.

89. Lau A, Wang S, Jiang J, et al. RIPK3-mediated necroptosis promotes donor kidney inflammatory injury and reduces allograft survival. Am J Transplant. 2013;13(11):2805-18.

90. Linkermann A, Bräsen JH, Himmerkus N, et al. Rip1 (receptorinteracting protein kinase 1) mediates necroptosis and contributes to renal ischemia/reperfusion injury. Kidney Int. 2012;81(8): 751-61.

91. Linkermann A, De Zen F, Weinberg J, Kunzendorf U, Krautwald S. Programmed necrosis in acute kidney injury. Nephrol Dial Transplant. 2012;27(9):3412-9.

92. Xu Y, Ma H, Shao J, et al. A Role for tubular necroptosis in cisplatin-induced AKI. J Am Soc Nephrol. 2015.

93. Linkermann A, Heller JO, Prokai A, et al. The RIP1-kinase inhibitor necrostatin-1 prevents osmotic nephrosis and contrast-induced AKI in mice. J Am Soc Nephrol. 2013;24(10):1545-57.

94. Takahashi N, Duprez L, Grootjans S, et al. Necrostatin-1 analogues: critical issues on the specificity, activity and in vivo use in experimental disease models. Cell Death Dis. 2012;3.

95. Vandenabeele P, Grootjans S, Callewaert N, Takahashi N. Necrostatin-1 blocks both RIPK1 and IDO: consequences for the study of cell death in experimental disease models. Cell Death Differ. 2013;20(2):185-7.

96. Edwards JR, Diamantakos EA, Peuler JD, Lamar PC, Prozialeck WC. A novel method for the evaluation of proximal tubule epithelial cellular necrosis in the intact rat kidney using ethidium homodimer. BMC Physiol. 2007;7.

97. Wang H, Sun L, Su L, et al. Mixed lineage kinase domain-like protein MLKL causes necrotic membrane disruption upon phosphorylation by RIP3. Mol Cell. 2014;54(1):133-46.

98. Northington FJ, Chavez-Valdez R, Graham EM, Razdan S, Gauda EB, Martin LJ. Necrostatin decreases oxidative damage, inflammation, and injury after neonatal HI. J Cereb Blood Flow Metab. 2011;31(1):178-89.

99. Smith CC, Davidson SM, Lim SY, Simpkin JC, Hothersall JS, Yellon DM. Necrostatin: a potentially novel cardioprotective agent? Cardiovasc Drugs Ther. 2007;21(4):227-33.

100. Liedtke C, Bangen JM, Freimuth J, et al. Loss of caspase-8 protects mice against inflammation-related hepatocarcinogenesis but induces non-apoptotic liver injury. Gastroenterology. 2011;141(6): 2176-87.

101. Cypel M, Rubacha M, Yeung J, et al. Normothermic ex vivo perfusion prevents lung injury compared to extended cold preservation for transplantation. Am J Transplant. 2009;9(10):2262-9.

102. Fischer S, Maclean AA, Liu M, et al. Dynamic changes in apoptotic and necrotic cell death correlate with severity of ischemiareperfusion injury in lung transplantation. Am J Respir Crit Care Med. 2000;162(5):1932-9.

103. Wu H, Chen G, Wyburn KR, et al. TLR4 activation mediates kidney ischemia/reperfusion injury. J Clin Invest. 2007;117(10): 2847-59.

104. Wu H, Ma J, Wang P, et al. HMGB1 contributes to kidney ischemia reperfusion injury. J Am Soc Nephrol. 2010;21(11):1878-90.

105. Zhang Z-X, Wang S, Huang X, et al. NK cells induce apoptosis in tubular epithelial cells and contribute to renal ischemiareperfusion injury. J Immunol. 2008;181(11):7489-98.

106. Lau A, Wang S, Liu W, Haig A, Zhang ZX, Jevnikar AM. Glycyrrhizic acid ameliorates HMGB1-mediated cell death and inflammation after renal ischemia reperfusion injury. Am J Nephrol. 2014;40(1):84-95.

107.• Zhao H, Ning J, Lemaire A, et al. Necroptosis and parthanatos are involved in remote lung injury after receiving ischemic renal allografts in rats. Kidney Int. 2015;87(4):738-48. The paper demonstrates the distant effect of $\mathrm{AKI}$ in inducing regulated necrosis in the lungs of transplant recipients.

108. Xu X, Chua KW, Chua CC, Liu CF, Hamdy RC, Chua BH. Synergistic protective effects of humanin and necrostatin-1 on hypoxia and ischemia/reperfusion injury. Brain Res. 2010;1355: 189-94.

109. Koshinuma S, Miyamae M, Kaneda K, Kotani J, Figueredo VM. Combination of necroptosis and apoptosis inhibition enhances cardioprotection against myocardial ischemia-reperfusion injury. J Anesth. 2014;28(2):235-41.

110. Pallet N, Dieudé M, Cailhier J, Hébert M. The molecular legacy of apoptosis in transplantation. Am J Transplant. 2012;12(6): 1378-84.

111. Ferguson TA, Choi J, Green DR. Armed response: how dying cells influence T-cell functions. Immunol Rev. 2011;241(1):77-88.

112. Kazama H, Ricci J-E, Herndon JM, Hoppe G, Green DR, Ferguson TA. Induction of immunological tolerance by apoptotic cells requires caspase-dependent oxidation of high-mobility group box-1 protein. Immunity. 2008;29(1):21-32.

113. Laplante P, Raymond M-A, Labelle A, Abe J-I, Iozzo RV, Hébert $\mathrm{M}-\mathrm{J}$. Perlecan proteolysis induces an alpha2betal integrin- and Src family kinase-dependent anti-apoptotic pathway in fibroblasts in the absence of focal adhesion kinase activation. J Biol Chem. 2006;281(41):30383-92. 
114. Soulez M, Sirois I, Brassard N, et al. Epidermal growth factor and perlecan fragments produced by apoptotic endothelial cells co-ordinately activate ERK1/2-dependent antiapoptotic pathways in mesenchymal stem cells. Stem Cells. 2010;28(4):810-20.

115. Du C, Guan Q, Yin Z, Masterson M, Zhong R, Jevnikar AM. Renal tubular epithelial cell apoptosis by Fas-FasL-dependent self-injury can augment renal allograft injury. Transplant Proc. 2003;35(7):2481-2.

116. Du C, Jiang J, Guan Q, et al. NOS2 (iNOS) deficiency in kidney donor accelerates allograft loss in a murine model. Am J Transplant. 2007;7(1):17-26.

117. Zhang Z, Zhu L, Quan D, et al. Pattern of liver, kidney, heart, and intestine allograft rejection in different mouse strain combinations. Transplantation. 1996;62(9):1267-72.

118. Zhang ZX, Huang X, Jiang J, et al. Natural killer cells mediate long-term kidney allograft injury. Transplantation. 2015.

119. Yang Y, Zhang ZX, Lian D, Haig A, Bhattacharjee RN, Jevnikar AM. IL-37 inhibits IL-18-induced tubular epithelial cell expression of pro-inflammatory cytokines and renal ischemia-reperfusion injury. Kidney Int. 2015;87(2): 396-408.

120. Jevnikar AM, Wuthrich RP, Brennan DC, Maslinski W, Glimcher LH, Rubin-Kelley VE. TNF-alpha is expressed on the surface of kidney proximal tubular cells. Transplant Proc. 1991;23(1 Pt 1): 231-2.
121. Shinozaki M, Hirahashi J, Lebedeva T, et al. IL-15, a survival factor for kidney epithelial cells, counteracts apoptosis and inflammation during nephritis. J Clin Invest. 2002;109(7):951-60.

122. Brouckaert G, Kalai M, Krysko DV, et al. Phagocytosis of necrotic cells by macrophages is phosphatidylserine dependent and does not induce inflammatory cytokine production. Mol Biol Cell. 2004;15(3):1089-100.

123. Lohmann C, Muschaweckh A, Kirschnek S, Jennen L, Wagner H, Hacker G. Induction of tumor cell apoptosis or necrosis by conditional expression of cell death proteins: analysis of cell death pathways and in vitro immune stimulatory potential. J Immunol. 2009;182(8):4538-46.

124. Hashmi SS, Ford M, Larsen C, Kirk A. Necrosis and immune activation: does tissue death alter acquired alloimmunity? J Am Coll Surg. 2013;7:329.

125. Upton JW, Kaiser WJ, Mocarski ES. DAI/ZBP1/DLM-1 complexes with RIP3 to mediate virus-induced programmed necrosis that is targeted by murine cytomegalovirus vIRA. Cell Host Microbe. 2012;11(3):290-7.

126. Upton JW, Chan FK. Staying alive: cell death in antiviral immunity. Mol Cell. 2014;54(2):273-80.

127. Omoto S, Guo H, Talekar GR, Roback L, Kaiser WJ, Mocarski E. Suppression of RIP3-dependent necroptosis by human cytomegalovirus. J Biol Chem. 2015.

128. Locke JE, Zachary AA, Warren DS, et al. Proinflammatory events are associated with significant increases in breadth and strength of HLA-specific antibody. Am J Transplant. 2009;9(9):2136-9. 